University of Nebraska - Lincoln

DigitalCommons@University of Nebraska - Lincoln

September 1995

\title{
Anisotropic anharmonicity of lattice and molecular vibrations of $1,2,4,5$ tetrabromobenzene determined by piezomodulated Raman spectroscopy
}

\author{
K.M. White \\ University of Nebraska - Lincoln \\ Craig J. Eckhardt \\ University of Nebraska - Lincoln, ceckhardt1@unl.edu
}

Follow this and additional works at: https://digitalcommons.unl.edu/chemistryeckhardt

Part of the Chemistry Commons

White, K.M. and Eckhardt, Craig J., "Anisotropic anharmonicity of lattice and molecular vibrations of 1,2,4,5 tetrabromobenzene determined by piezomodulated Raman spectroscopy" (1995). Craig J. Eckhardt Publications. 13.

https://digitalcommons.unl.edu/chemistryeckhardt/13

This Article is brought to you for free and open access by the Published Research - Department of Chemistry at DigitalCommons@University of Nebraska - Lincoln. It has been accepted for inclusion in Craig J. Eckhardt Publications by an authorized administrator of DigitalCommons@University of Nebraska - Lincoln. 


\title{
Anisotropic anharmonicity of lattice and molecular vibrations of $1,2,4,5-$ tetrabromobenzene determined by piezomodulated Raman spectroscopy
}

\author{
K. M. White ${ }^{a)}$ and C. J. Eckhardt ${ }^{\text {b) }}$ \\ Department of Chemistry, University of Nebraska-Lincoln, Lincoln, Nebraska 68588-0304
}

(Received 7 April 1995; accepted 23 May 1995)

\begin{abstract}
Strain-induced coupling constants for the anharmonicity of Raman-active lattice and certain molecular modes of vibration in 1,2,4,5-tetrabromobenzene (TBB) crystals have been determined using piezomodulated Raman spectroscopy. These constants, which are directly related to the first anharmonic term in the potential energy expansion for lattice dynamical calculations, are a quantitative measurement of the modal anharmonicities in the TBB molecular crystal. Application of uniaxial stress in the experiments permits the anisotropy of the anharmonicity to be determined as well as its magnitude. The TBB lattice modes are significantly coupled by the induced strains and the effects of coupling were observed to be dependent on the direction and symmetry of the strains. The molecular modes investigated were, by comparison, less coupled by the acoustic phonons and generally exhibited less anharmonic response with increasing frequency. (C) 1995 American Institute of Physics.
\end{abstract}

\section{INTRODUCTION}

The study of intermolecular potential energy in molecular crystals is vital to an understanding of this class of solids. The benefits of such research in the crystalline state are manifold, since along with the influence they exert on crystal packing, intermolecular interactions contribute to the mechanical, optical, electrical, and thermal properties of solids.

Investigations of the potential energy of molecular crystals by both theoretical and experimental means comprise an active area of solid state research. ${ }^{1}$ Perhaps the most effective current approach for calculation of the lattice energy has involved the use of the parametrized Buckingham or Lennard-Jones potentials. $^{2}$ These calculations have often complemented experimental studies that have focused on the lattice dynamics that are governed by the potential energy. Lattice vibrational energies of a number of molecular crystals have been measured by Raman spectroscopy and in several cases the symmetries of the modes have been assigned. A comparison of calculated and observed results shows that while the crystal potentials derived have successfully predicted frequencies and symmetries of lattice modes in some systems, they are quite inadequate in others.

The need to improve the functions and models used for the potential energies of molecular crystals is evident. One important aspect that is experimentally accessible is the anharmonic contribution to the potential which is normally neglected when applying the harmonic approximation in studies of lattice dynamics. ${ }^{3,4}$ Anharmonicity is manifested by shifts of phonon frequencies from harmonic values and by the finite lifetime of phonon states. It is also responsible for thermal expansion and stability of a crystal undergoing a phase transition and plays an important role in thermal and electrical conductivity. ${ }^{5}$ This coupling between vibrational modes in molecular crystals has been studied by various ex-

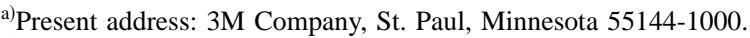

b) Author to whom correspondence should be addressed.
}

perimental methods, including measurements of Raman linewidths and frequency shifts as a function of temperature and pressure. ${ }^{6-8}$ However, the anistropy of the anharmonic crystal potential has not been investigated extensively for molecular crystals.

The theoretical ${ }^{9}$ and experimental ${ }^{10,11}$ development of piezomodulated Raman (PR) spectroscopy has demonstrated that this technique directly probes anharmonicity of vibrational modes in crystals. Specifically, PR spectroscopy probes the acoustic phonon mediated coupling of optical modes and measures the corresponding strain-induced coupling parameters. While the usefulness of such measurements in understanding solids has been proposed, ${ }^{12}$ the coupling parameters are still largely unknown, particularly for molecular crystals. A significant advantage of PR spectroscopy over Raman measurements on strained molecular crystals is that the experiment makes use of a small uniaxial stress rather than a large hydrostatic one. This enables the elastic regime of the crystal to be probed and allows the extraction of directional information regarding the crystal potential energy.

It has been demonstrated that PR spectroscopy displays a sensitivity to structure that is not available in more traditional measurements. A PR study of 1,2,4,5tetrabromobenzene reported that the motions of the molecules involved in the vibrational modes of the crystal could be determined by observing how the frequencies of the modes change under stresses applied to the crystal in different directions. ${ }^{11}$ A recent study of phenothiazine further established the efficacy of PR spectroscopy by providing an understanding of the complicated phase transition behavior of that system. ${ }^{13}$

Previously, a detailed study of the displacive phase transition in 1,2,4,5-tetrabromobenzene (TBB) by Raman spectroscopy has been reported. ${ }^{14}$ Although this work permitted the assignment of observed modes, it did not unambiguously 
identify the soft modes nor the associated anharmonicities. In this paper, the study of strain-induced coupling in the lattice dynamics of TBB by means of piezomodulated Raman spectroscopy is reported. By obtaining an unambiguous PR response in a molecular crystal, an interpretation based on the theory of Luty and Eckhardt ${ }^{9}$ is used to quantify the anharmonic character of the various vibrational modes through the derivation of their respective coupling parameters.

TBB was chosen for this study for a number of reasons. First, TBB exhibits high compressibility. Thus, strains that are sufficiently large for detection of PR signals can be easily produced in the crystalline samples. Second, because it has been shown that a distinct separation between the external and internal modes in TBB exists, ${ }^{14}$ a determination of the extent of strain-induced coupling exhibited by its lattice vibrations can be compared to that for its intramolecular modes. This provides a useful test of the concept that molecular vibrations, being less coupled to the crystal lattice, are more harmonic than the lower frequency lattice modes. Finally, the TBB crystal is also known to undergo a structural phase transition just above room temperature. ${ }^{15-17}$ As a result, identification of highly anharmonic lattice modes via PR spectroscopy may be expected to help identify those vibrations that play the most significant roles in the mechanism of the phase change.

\section{THEORETICAL BACKGROUND}

A foundation for the study of anharmonicity in crystals using piezomodulated Raman spectroscopy has been established in the theoretical work of Luty and Eckhardt. ${ }^{9}$ The basic idea is that a periodic stress applied to a crystal enhances phonon-phonon coupling that is mediated by the acoustic phonons associated with the essentially static (on the time scale of the interactions) strain created by the piezomodulation. The presence of this coupling in the lattice results in frequency shifts of the vibrational modes when the crystal is under stress. The magnitude of the shifts is dependent on the strain and the extent of strain-induced coupling exhibited by the various modes. The PR spectra obtained represent the difference between Raman responses at the extremes of extension and compression of the crystal and are thus a measure of anharmonicity in the crystal vibrations.

The derivation of this theory requires the formulation of a renormalized Hamiltonian that describes the energy of a strained lattice. For a lattice with unit cell volume $v$ that experiences strain $e_{\alpha \beta}$, the effective Hamiltonian is

$$
\begin{aligned}
\mathscr{H}= & \mathscr{H}_{0}+\frac{1}{2} v \sum_{\alpha \beta \gamma \delta} C_{\alpha \beta \gamma \delta} e_{\alpha \beta} e_{\gamma \delta} \\
& +\frac{1}{2} \sum_{j j^{\prime}}\left(\omega_{0 j}^{2} \delta_{j j^{\prime}}+\sum_{\alpha \beta} \phi_{\alpha \beta, j j^{\prime}} e_{\alpha \beta}\right) Q_{j} Q_{j^{\prime}},
\end{aligned}
$$

where $\mathscr{H}_{0}$ is the Hamiltonian for the undeformed lattice, $C_{\alpha \beta \gamma \delta}$ is the effective elastic constant, $Q_{j}$ and $\omega_{0 j}$ represent the normal coordinate and fundamental frequency of vibrational mode $j$, respectively, and the coupling between the optical phonons due to strain is given by $\phi_{\alpha \beta, j j^{\prime}}$.
The second and third terms of Eq. (1) represent the contributions to the crystal energy arising from the presence of strains and vibrations, respectively, in the lattice. With the exception of the effects of internal strain included in the effective elastic constant $C_{\alpha \beta \gamma \delta}$, these two terms only involve harmonic interactions in the crystal as evidenced by their resemblance to Hooke's law. On the other hand, the last term in Eq. (1) arises from strain-induced coupling between vibrational modes in the crystal. The contribution of this term to the energy of the lattice depends not only on the amount and type of strain in the crystal, but also on the magnitude of the coupling constant, $\phi_{\alpha \beta, j j^{\prime}}$. Cowley ${ }^{12}$ has demonstrated the relationship between $\phi_{\alpha \beta, j j^{\prime}}$ and the first anharmonic term in the potential energy expansion employed in the vibrational analysis of a crystal. The determination of $\phi_{\alpha \beta, j j^{\prime}}$ for a particular mode based on its PR spectrum will therefore not only provide a measure of anharmonicity for that mode, but the directional dependence of the response can also reveal information about the anisotropy of the lattice potential energy.

The value of $\phi_{\alpha \beta, j j^{\prime}}$ is manifested by the shift in vibrational mode frequencies in the strained crystal, as derived from the associated term

$$
\frac{1}{2} \sum_{j j^{\prime}}\left(\omega_{0}^{2} \delta_{j j^{\prime}}+\sum_{\alpha \beta} \phi_{\alpha \beta, j j^{\prime}} e_{\alpha \beta}\right) Q_{j} Q_{j^{\prime}}
$$

of Eq. (1). However, given the size of the strains used in the experiments, these frequency shifts are quite small and therefore must be determined indirectly by measuring the intensity difference between Raman bands for the extended and compressed crystal as a function of frequency. Consequently, the data analysis requires that an expression for the intensity of the PR signal be formulated as a strain derivative of the Raman response which shows a dependence on $\phi_{\alpha \beta, j j^{\prime}}$.

Definition of the complex electronic susceptibility in terms of the renormalized coordinates and representation of the Raman intensity by correlation of the susceptibility leads to an expression for the band shape of the inelastically scattered light affected by the strain. For the one-phonon oscillator approximation with an associated susceptibility, $\chi$, the band shape of the PR signal corresponding to induced strain $e_{\alpha \beta}$ is given by ${ }^{9}$

$$
\frac{\partial \chi^{\prime \prime}(\omega)}{\partial e_{\alpha \beta}} \cdot \Delta e_{\alpha \beta}=-\Delta e_{\alpha \beta} \cdot \operatorname{Im}\left[\chi^{0}(\omega) \phi_{\alpha \beta} \chi^{0}(\omega)\right]
$$

for sufficiently small strains. Here, $\chi^{0}$ is the complex susceptibility when strain is absent. The imaginary part of the susceptibility, $\chi^{\prime \prime}$, describes the corresponding Raman band shape. For the PR experiment, $\Delta e$ for any specified strain is defined as

$$
\Delta e=e_{\mathrm{ext}}-e_{\mathrm{comp}},
$$

which is the difference between the strains produced during the extensive and compressive portions of the stress cycle. Convention dictates ${ }^{18}$ that $e_{\mathrm{ext}}>0$ and $e_{\text {comp }}<0$ for the strains parallel to the respectively applied stresses. The crucial result of the analysis is that the strength of the response will depend on the amount of coupling between the modes 


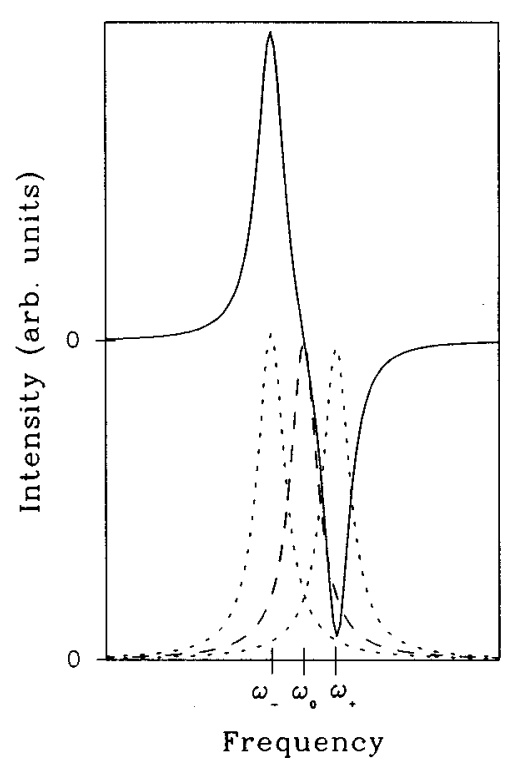

FIG. 1. Hypothetical piezomodulated Raman response (solid line) corresponding to a mode with a Raman resonance (dashed line) at $\omega_{0}$. The dotted lines represent Raman bands for this mode at the extrema of the applied tensile and compressive stresses.

which is induced by the strain. An ideally harmonic mode will give no piezomodulated Raman signal since the coupling constant, $\phi_{\alpha \beta}$, would be zero.

Evaluation of $\phi_{\alpha \beta}$ is accomplished through a curve fitting procedure detailed elsewhere. ${ }^{13}$ From Eq. (2), the corresponding expression for the piezomodulated Raman response is given by ${ }^{9}$

$$
\begin{aligned}
\Delta I(\omega) & =\frac{\partial I(\omega)}{\partial e_{\alpha \beta}} \cdot \Delta e_{\alpha \beta} \\
& =-\Delta e_{\alpha \beta} \cdot \frac{2 \phi_{\alpha \beta} A \omega \gamma\left(\omega_{0}^{2}-\omega^{2}\right)}{\left[\left(\omega_{0}^{2}-\omega^{2}\right)^{2}-\omega^{2} \gamma^{2}\right]^{2}},
\end{aligned}
$$

where $\gamma$ is the full width at half height of the corresponding Raman band and $A$ is a factor that accounts for its intensity, $I$. The coupling constant for a given vibrational mode is determined by first fitting the experimental Raman band to obtain $\omega_{0}, \gamma$, and $A$ and then inserting these values into Eq. (4) to fit the PR curve and obtain $\Delta e_{\alpha \beta} \cdot \phi_{\alpha \beta}$. Dividing this expression by the strain will produce the desired constant, $\phi_{\alpha \beta}$. Under the one phonon approximation, the effective $\phi_{\alpha \beta}$ thus obtained for a given lattice mode is a measure of all coupling to that mode mediated by strain $e_{\alpha \beta}$.

In light of the above theoretical discussion, the PR response can be described by

$$
\Delta I(\omega)=I_{\omega_{\text {ext }}}(\omega)-I_{\omega_{\text {comp }}}(\omega),
$$

where $I_{\omega_{\text {ext }}}$ and $I_{\omega_{\text {comp }}}$ represent the Raman response of a vibrational mode whose resonance frequency shifts to $\omega_{\text {ext }}$ when the crystal is under tensile stress and $\omega_{\text {comp }}$ when it is under compressive stress (see Fig. 1). It can be shown by lengthy but straightforward algebra that for small strains, Eq.

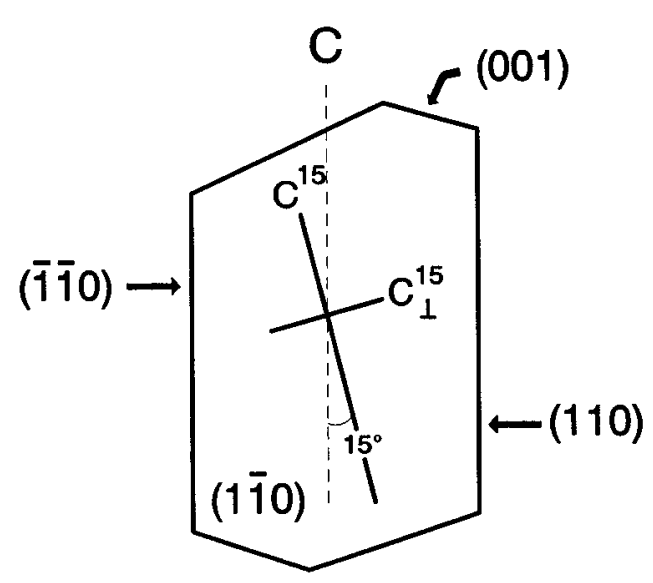

FIG. 2. Crystal habit of the beta phase of TBB. The axes $c^{15}$ and $c_{\perp}^{15}$ indicate the principal directions for the form $\{110\}$.

(5) is identical to the strain derivative in Eq. (4), assuming from the one phonon oscillator approximation of Luty and Eckhardt ${ }^{9}$ that

$$
\omega_{\mathrm{ext}}^{2}=\omega_{0}^{2}+\phi_{\alpha \beta} e_{\mathrm{ext}}
$$

and

$$
\omega_{\text {comp }}^{2}=\omega_{0}^{2}+\phi_{\alpha \beta} \cdot e_{\text {comp }}=\omega_{0}^{2}+\phi_{\alpha \beta} \cdot\left(-e_{\text {ext }}\right),
$$

where $\omega_{0}$ is the frequency of the mode in an unstressed crystal. If $\phi_{\alpha \beta}<0$, then $\omega_{\text {ext }}$ can be denoted as $\omega_{-}$, which is less than $\omega_{0}$, and $\omega_{\text {comp }}$ denoted as $\omega_{+}$, which is greater than $\omega_{0}$.

\section{EXPERIMENT}

TBB obtained from Aldrich Chemical was purified by vacuum sublimation and recrystallized from chloroform yielding single crystals in the room temperature beta phase. The crystals, which have space group symmetry $P 2_{1} / a\left({ }^{5} C_{2 h}\right)$, are columnar along $c$ with form $\{110\}$ (Fig. 2). The crystal habit and optical properties of the beta phase have been described previously. ${ }^{14}$

The TBB crystal was mounted between a stationary stainless steel bar and a lead zirconate titanate (PZT) piezoceramic bar which was clamped at the opposite end. The sample was fixed in the anvil using a rapid-setting epoxy (Devcon 5-Minute). The crystal was oriented for application of uniaxial stress in either the [110]* or [001] direction, where [110]* is defined to be normal to the (110) crystal face. The entire assembly was mounted in a $90^{\circ}$ scattering geometry. Application of a sinusoidal voltage $(200 \mathrm{~Hz})$ across the PZT causes alternate longitudinal contractions and expansions, thereby applying tensile and compressive stresses to the crystal.

Since the TBB crystal was found to be extremely compressible compared to the PZT, the change in length of the unclamped portion of the PZT $\left(\Delta L_{\mathrm{PZT}}\right)$ was assumed to be the same as that for a free PZT bar,

$$
\Delta L_{\mathrm{PZT}}=d_{31}\left(\frac{V}{t}\right) L_{\mathrm{PZT}}
$$


TABLE I. Polarization and stress configurations used in the piezomodulated Raman spectra of TBB.

\begin{tabular}{|c|c|c|c|c|c|}
\hline $\begin{array}{r}\text { Designated } \\
\text { scattering } \\
\text { geometry }\end{array}$ & $\begin{array}{c}\text { Stress } \\
\text { direction }\end{array}$ & $\begin{array}{l}\text { Incident beam } \\
\text { direction }\end{array}$ & $\begin{array}{c}\text { Incident beam } \\
\text { polarization }\end{array}$ & $\begin{array}{l}\text { Scattered light } \\
\text { direction }\end{array}$ & $\begin{array}{c}\text { Scattered light } \\
\text { polarization }\end{array}$ \\
\hline$A$ & {$[110]^{*}$} & $c$ & $b$ & Normal to $(1 \overline{1} 0)$ & $c^{15}$ \\
\hline$B$ & {$[110]^{*}$} & $c$ & $a^{*}$ & Normal to $(1 \overline{1} 0)$ & $c^{15}$ \\
\hline C & {$[110]^{*}$} & $c$ & $b$ & Normal to $(1 \overline{1} 0)$ & $c_{\perp}^{15}$ \\
\hline$D$ & {$[001]$} & Normal to $(\overline{1} 10)$ & $c^{15}$ & Normal to $(\overline{1} 10)$ & $c^{\frac{1}{15}}$ \\
\hline$E$ & [001] & Normal to $(\overline{1} 10)$ & $c^{15}$ & Normal to $(\overline{1} 10)$ & $c_{\perp}^{15}$ \\
\hline
\end{tabular}

where $d_{31}$ is the piezoelectric coefficient that relates the voltage $V$ applied across a PZT having a thickness $t$ to its induced strain. Thus, the strain induced in the crystal along the stress direction can be calculated as

$$
e_{\mathrm{xtal}}=\Delta l_{\mathrm{xtal}} / l_{\mathrm{xtal}},
$$

where, owing to the experimental configuration, $\Delta L_{\mathrm{PZT}}$ $=-l_{\mathrm{xtal}}$. In this manner, the strains induced in the TBB crystals were found to be on the order of 0.001 for both the $[110]^{*}$ and [001] stress configurations.

Since the intensity of the piezomodulated Raman response for a given vibrational mode depends in part on its Raman intensity, a Raman spectrum was measured before each PR spectrum using the same scattering geometry. Consequently, relative intensities obtained from the two types of spectra could be used for data analysis. The five different polarization and stress configurations employed in the various experiments are listed in Table I and each is designated with a letter for reference throughout the paper.

Polarized PR spectra of TBB were measured in the lattice mode region from 8 to $60 \mathrm{~cm}^{-1}$ for stress applied along the $[110]^{*}$ or $[001]$ directions. Additional spectra that employed only the $[110]^{*}$ stress were recorded for various intramolecular vibrations. Measurements were obtained with the $5145 \AA$ line of an argon ion laser operating at $55 \mathrm{~mW}$. The incident beam was chopped during Raman measurements. For both Raman and PR spectra, modulation of the scattered light intensity was detected using synchronous lock-in amplification. Data points were recorded at $0.5 \mathrm{~cm}^{-1}$ intervals with a bandpass of $1.4 \mathrm{~cm}^{-1}$. Instrumentation used to analyze and detect the scattered light is described elsewhere. ${ }^{10,19}$

\section{RESULTS}

\section{A. Lattice modes}

\section{1. $[110]^{*}$ stress}

Piezomodulated Raman spectra of TBB that employed a $[110]^{*}$ stress are shown in Fig. 3 along with their corresponding Raman spectra. The Raman and PR intensity scales shown in a given graph indicate the actual relative intensities obtained for the two types of spectra.

The Raman spectrum obtained for the $A$ scattering geometry contains bands assigned ${ }^{14}$ to the $B_{g}$ lattice vibrations at $21.5,43$, and $47.5 \mathrm{~cm}^{-1}$ as well as the $15.5 \mathrm{~cm}^{-1} A_{g}$ mode. Of these modes, the one at $21.5 \mathrm{~cm}^{-1}$ is seen to have the strongest piezomodulated Raman response. Its band shape is similar in appearance to the first derivative with respect to frequency of its corresponding Raman band, with the band going positive and then negative as one scans the band from low to high energy. For purposes of discussion, this type of PR band shape will be referred to as a"positive" band.

The other bands observed in the Raman spectrum for the $A$ configuration also have corresponding structure in the PR spectrum. However, the PR bands of these lattice vibrations are relatively weak compared to their Raman bands. In addition, the PR band of these lattice modes are inverted. Such bands will be termed "negative" bands. Their appearance

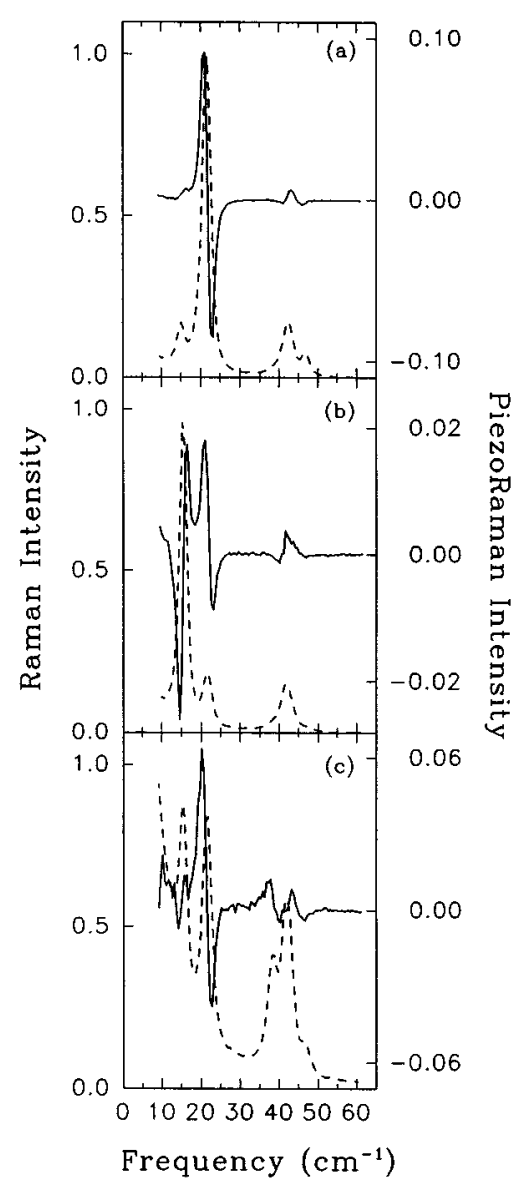

FIG. 3. Room temperature Raman (dashed line) and piezomodulated Raman (solid line) spectra of lattice modes in the beta phase of TBB for stress applied along $[110]^{*}$. Scattering geometries employed were (a) $A$, (b) $B$, and (c) $C$. Relative intensities for each Raman and PR pair of spectra are indicated. 
indicates that the lattice vibrations in TBB with which they correspond respond differently to a given applied stress than do those vibrations associated with a "positive" band.

Another interesting aspect of the PR spectrum for configuration $A$ is the symmetry (or lack of it) of each band about the line $\Delta I(\omega)=0$. The band at $21.5 \mathrm{~cm}^{-1}$ is seen to be neatly symmetric and the band at $15.5 \mathrm{~cm}^{-1}$, which is riding on the positive shoulder of the $21.5 \mathrm{~cm}^{-1}$ band, also appears to exhibit nearly equal positive and negative excursions. However, the bands observed just above $40 \mathrm{~cm}^{-1}$ are quite asymmetric. Indeed, while both bands are definitely negative, the PR response for the $47.5 \mathrm{~cm}^{-1}$ mode appears to be lacking the positive-going portion of its band.

The negative band is observed more clearly in the PR spectrum for the $B$ scattering geometry. In this configuration, the 15.5 and $41.5 \mathrm{~cm}^{-1} A_{g}$ lattice modes ${ }^{14}$ are most prominent in the Raman spectrum, although $B_{g}$ bands at 21.5 and $47.5 \mathrm{~cm}^{-1}$ are also observed. The PR spectrum shows a significant negative type response for the $15.5 \mathrm{~cm}^{-1}$ lattice vibration. However, the ratio of its intensity to that of its Raman band is still smaller than the ratio obtained for the PR and Raman bands of the $21.5 \mathrm{~cm}^{-1}$ mode.

A negative band is also observed in the $B$ PR spectrum near $41.5 \mathrm{~cm}^{-1}$. A comparison of the $A$ and $B$ PR spectra reveals that this band occurs at lower energy than the similarly shaped band discussed previously which corresponded to the $43 \mathrm{~cm}^{-1}$ mode. The weak, negative-going lobe seen in the $B$ PR spectrum just to the higher energy side of the 41.5 $\mathrm{cm}^{-1}$ structure is located at the same frequency as the band observed in the $A$ spectrum which also only showed a negative excursion. Both apparently arise from the $47.5 \mathrm{~cm}^{-1}$ lattice vibration. Asymmetry of the PR bands in the $B$ spectrum is also noticeable.

In the $C$ scattering geometry, both the $A_{g}$ and $B_{g}$ lattice modes in TBB exhibit significant intensities in the Raman spectrum. Moreover, this is the only spectrum of the three considered thus far in which the $39 \mathrm{~cm}^{-1} A_{g}$ lattice mode ${ }^{14}$ is clearly resolved. In the piezomodulated Raman spectrum, this mode gives rise to a positive PR band along with the still dominant $21.5 \mathrm{~cm}^{-1} B_{g}$ lattice vibration.

The remaining structure in the $C$ PR spectrum is consistent with that obtained in the $A$ and $B$ configurations. In the region above $40 \mathrm{~cm}^{-1}$, the bands are neatly identical in both frequency and shape to those observed in the $A$ scattering geometry where the 43 and $47.5 \mathrm{~cm}^{-1}$ bands were detected in the Raman spectrum. However, since in the $C$ Raman spectrum it is not possible to resolve the $41.5 \mathrm{~cm}^{-1} A_{g}$ mode from the $43 \mathrm{~cm}^{-1} B_{g}$ mode, it is difficult to determine to what extent the $41.5 \mathrm{~cm}^{-1}$ mode contributes to the PR intensity in this energy region for the $C$ experimental configuration.

The PR spectral frequencies and band shapes observed for the $A, B$, and $C$ scattering geometries were not only consistent from geometry to geometry, but were also reproducible for different crystal samples. The magnitude of the PR responses, however, were found to be extremely sensitive to the quality of the crystal. As a result, the PR relative intensities varied somewhat from crystal to crystal. The PR spectra shown in Fig. 3 were taken from crystals producing

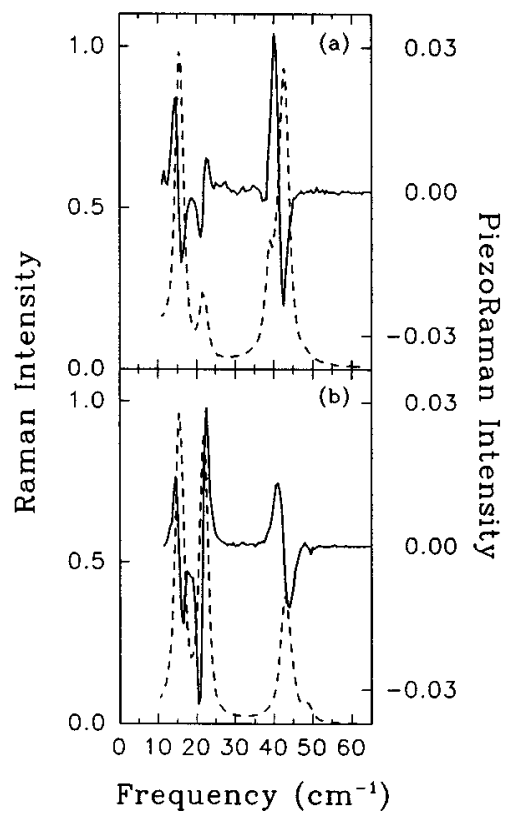

FIG. 4. Room temperature Raman (dashed line) and piezomodulated Raman (solid line) spectra of lattice modes in the beta phase of TBB for stress applied along [001]. Scattering geometries employed were (a) $D$ and (b) $E$. Relative intensities for each Raman and PR pair of spectra are indicated.

the largest PR responses. The reason for this will be considered in greater detail in the Discussion.

This sensitivity to crystal quality also had an apparent effect on the PR band shape observed around $42 \mathrm{~cm}^{-1}$ in the $C$ scattering geometry. In $C$ PR spectra measured from two different crystals, the band near $42 \mathrm{~cm}^{-1}$ was negative in one spectrum, but positive in the other. This "change of phase" was observed in more than one crystal but only for this particular band in this specific scattering geometry.

\section{2. [001] stress}

The piezomodulated Raman spectra obtained for stresses applied along the $c$ axis of the crystal are found in Fig. 4. These spectra employed in $D$ and $E$ scattering geometries and their corresponding Raman spectra are also shown in the figure.

In the $D$ scattering geometry, the $A_{g}$ lattice modes at $15.5,39$, and $41.5 \mathrm{~cm}^{-1}$ dominate the Raman spectrum with a weak appearance of the $21.5 \mathrm{~cm}^{-1} B_{g}$ band. Examination of the $D$ PR spectrum reveals a dramatic change in the phases of the lattice mode PR bands from those obtained when the [110]* stress was applied. For each of the lattice vibrations observed in the direct Raman spectrum, the sense of its corresponding PR band has reversed; bands that were positive in the $[110]^{*}$ stress experiments are found to be negative in the [001] stress spectra, and vice versa. Recognizing that the positive excursion of the $39 \mathrm{~cm}^{-1} \mathrm{PR}$ negative band is buried under the positive portion of the $41.5 \mathrm{~cm}^{-1}$ positive band, the PR bands in the $D$ spectrum are also observed to be quite symmetric about zero on the ordinate scale. 


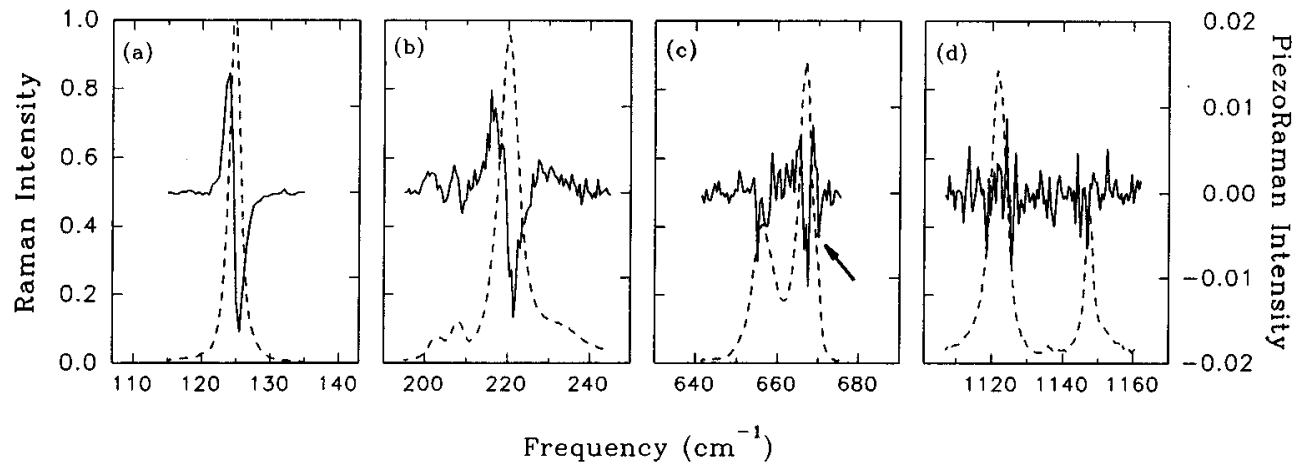

FIG. 5. Room temperature Raman (dashed line) and piezomodulated Raman (solid line) spectra of molecular modes in the beta phase of TBB for stress applied along [110]*. The scattering geometry employed was $C$. Relative intensities for each Raman and PR pair of spectra are indicated.

The Raman spectrum in the $E$ scattering geometry contains bands for the $21.5,43$, and $47.5 \mathrm{~cm}^{-1} B_{g}$ modes along with a very intense band for the $15.5 \mathrm{~cm}^{-1} A_{g}$ lattice vibration. Once again, each of these modes gives rise to structure in the PR spectrum and the phases of all of the PR bands are reversed from the results observed in the $[110]^{*}$ stress spectra. Nearly equal excursions about zero are also seen in each of the bands. This result is especially noteworthy for the band above $40 \mathrm{~cm}^{-1}$, since in this region the bands were found to be quite asymmetric in the [110]* stress spectra.

\section{B. Molecular modes}

A number of the intramolecular vibrational modes in TBB were also studied using piezomodulated Raman spectroscopy. Determination of symmetry assignments for these Raman active modes was made and will be published elsewhere. ${ }^{20}$ The majority of modes examined had $a_{g}$ symmetry, generally resulting in higher scattering intensities that aided the detection of their PR signals. Each of the PR spectra were measured using the $C$ scattering geometry with the stress applied along [110]*.

The Raman and PR spectra of the $125 \mathrm{~cm}^{-1} a_{g}$ molecular vibration are displayed in Fig. 5(a). An isolated, positive band having a nearly symmetric line shape is observed in the PR spectrum.

The Raman spectrum in the $200-240 \mathrm{~cm}^{-1}$ energy region plotted in Fig. 5(b) contains bands at 203, 208, and 220 $\mathrm{cm}^{-1}$ assigned as $b_{2 g}, b_{3 g}$, and $a_{g}$ intramolecular modes, respectively. In addition, a broad, featureless shoulder of unknown origin is observed on the high energy side of the 220 $\mathrm{cm}^{-1}$ band. In the corresponding PR spectrum, each of the fundamental vibrational modes is found to give rise to positive, derivativelike bands. The high energy shoulder also has corresponding structure in the PR spectrum, but it involves only a positive excursion in the region of $230 \mathrm{~cm}^{-1}$.

In Fig. 5(c) the Raman and PR spectra of TBB from 643 to $677 \mathrm{~cm}^{-1}$ are shown together. The intramolecular fundamental vibrations displaying Raman activity in this energy region include the $667 \mathrm{~cm}^{-1} a_{g}$ mode and the $670 \mathrm{~cm}^{-1}$ $b_{2 g}$ mode which is assigned to the very weak shoulder on the high energy side of the $667 \mathrm{~cm}^{-1}$ peak. The combination band at $657 \mathrm{~cm}^{-1}$, having a comparatively large intensity ascribed to a Fermi resonance interaction with the $667 \mathrm{~cm}^{-1}$ fundamental, ${ }^{20}$ is also observed in the Raman spectrum.

In the piezomodulated Raman spectrum for the 643-677 $\mathrm{cm}^{-1}$ interval, it can be seen that the PR response has diminished to the point that it is only just observable above the level of the noise. Nevertheless, some unusual features can still be distinguished. The most striking is the similarity of the PR band shape in the region near $667 \mathrm{~cm}^{-1}$ to a second derivative shape in contrast to the expected first derivative response. Also notable is the structure in the PR spectrum (see arrow) that has its origin in the weak $670 \mathrm{~cm}^{-1}$ shoulder seen in the Raman spectrum. The PR response near 657 $\mathrm{cm}^{-1}$ has a more complicated band shape.

The last intramolecular vibrational energy interval to be studied in TBB using piezomodulated Raman spectroscopy spanned $1105-1160 \mathrm{~cm}^{-1}$. The Raman and PR spectra are found in Fig. 5(d). In the Raman spectrum, the $1122 \mathrm{~cm}^{-1}$ $a_{g}$ fundamental and the overtone at $1145 \mathrm{~cm}^{-1}$ have been assigned to the strongest peaks recorded in this energy interval and additional, weak structure that is not yet assigned is observed near 1135 and $1150 \mathrm{~cm}^{-1}$. The PR spectrum does not provide any interpretable structure for these modes since the response is at the level of the noise.

\section{DISCUSSION}

The analysis of the piezomodulated Raman spectra of both the lattice and molecular vibrational modes in TBB focuses on several different features of the measured bands. The band shapes, the phases of the derivativelike bands, and the intensities of the bands relative to their corresponding Raman intensities must all be considered as each of these features provides important information about coupling of the vibrational modes and how it is manifested in the potential energy of the crystal.

\section{A. Shape and phase of the PR band}

The band shape of the piezomodulated Raman signal is similar, but not equivalent to the frequency derivative of the Raman band itself. To emphasize the point that PR spectra are not simply frequency derivatives of direct Raman spectra, the PR spectrum from Fig. 3(b) has been replotted in Fig. 6 along with the calculated first-frequency derivative of its 


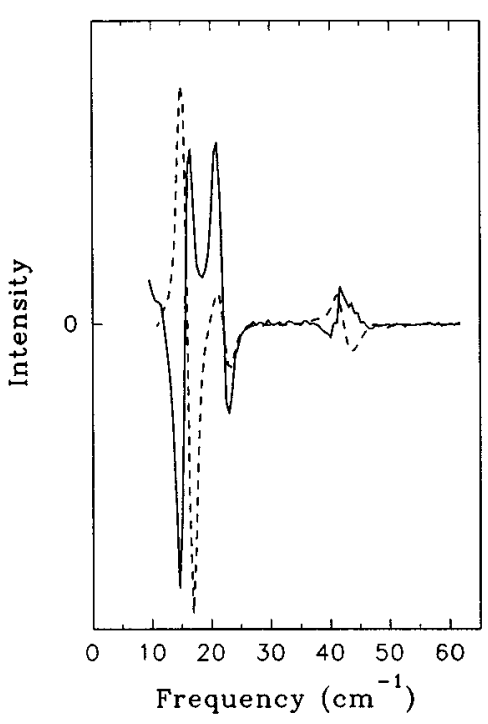

FIG. 6. Comparison of the PR spectrum (solid line) and the calculated frequency derivative (dashed line) of the Raman spectrum shown in Fig. 3 (b). Scaling is arbitrary.

corresponding experimental Raman spectrum. The amplitude of the derivative curve has been arbitrarily adjusted for purposes of comparison. Two significant differences are observed. First, the phases of the bands in the PR spectra are not always positive, as is the case for every band in the calculated frequency derivative. Second, the intensities of the PR bands relative to each other within the spectrum are not the same as those seen in the frequency derivative. Both of these features, which are discussed in subsequent sections, indicate that information in addition to the higher resolution typically obtained in frequency-modulated Raman spectra can be gained from the piezomodulated Raman spectra.

For the most part, the PR spectra of TBB found in Figs. 3-5 exhibit derivativelike band shapes of the type predicted by theory. They display both positive and negative excursions and are nearly symmetrical about the zero response line. It has been noted, however, that the PR bands in the lattice mode region between 40 and $50 \mathrm{~cm}^{-1}$ showed a significant departure from symmetry about the zero response line when the stress was applied along [110]*. The reason for this is not fully understood, although the close proximity of four of the lattice vibrations of TBB in this energy range may be part of the answer. Strong coupling among these modes may lead to some effects not accounted for in the current piezomodulated Raman theory or by the approximations employed.

Another PR band displaying an unexpected response is that belonging to the $667 \mathrm{~cm}^{-1}$ intramolecular vibration which involves a totally symmetric, in-plane ring deformation and bromine stretch. ${ }^{20}$ One of the measured PR spectra is shown in Fig. 7 along with the calculated first and second frequency derivatives of the experimental Raman band. The resemblance of the PR band to a second derivative shape is quite remarkable but may be fortuitous. Nevertheless, the band shape definitely does not possess a first derivativelike shape. It is possible that it derives from two first derivative

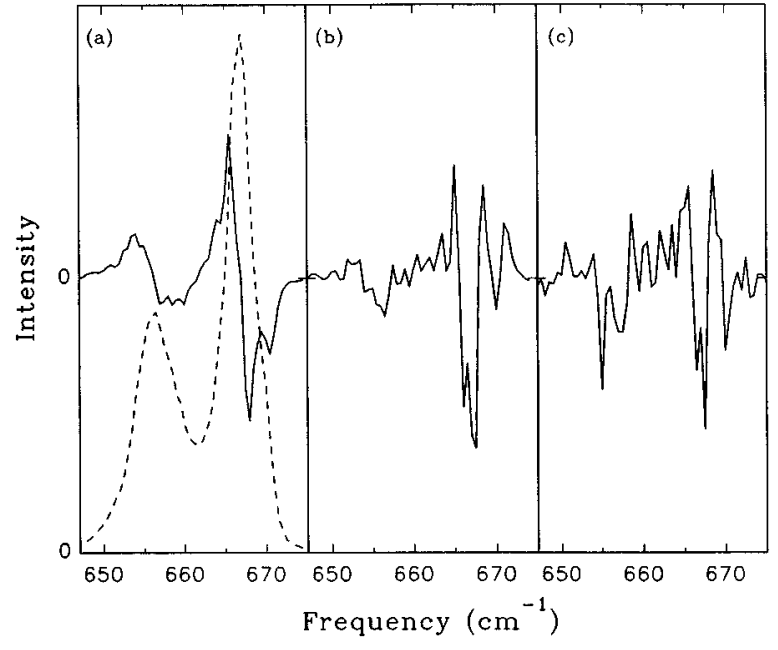

FIG. 7. Calculated (a) first and (b) second derivatives (solid line) of the measured Raman bands (dashed line) corresponding to molecular modes in TBB between 647 and $677 \mathrm{~cm}^{-1}$. (c) The PR spectrum measured from TBB in this frequency range.

type bands of opposite phase that overlap to give appearance of a second derivative type spectrum. The interactions here are obviously complicated and may stem from the Fermi resonance that is attributed to this band. Such an assignment implies that intensity from the nearby combination band as well as the $667 \mathrm{~cm}^{-1}$ fundamental is involved in the scattering response at this frequency. ${ }^{21}$

The use of uniaxial stresses in the PR experiments allows information to be gained from the phases of the observed bands. Stresses were applied such that a positive band corresponds to a mode in which a compressive stress increases the vibrational frequency and a tensile stress decreases it. Conversely, a mode giving rise to a negative band undergoes a decrease in frequency under compressive stress and an increase under tensile stress. Hence, as can be seen from the PR spectra in Figs. 3 and 4, the 21.5 and $39 \mathrm{~cm}^{-1}$ lattice modes increase in frequency under compressive stress in the [110]* direction and decrease for compressive stress along [001]. The remaining Raman-active lattice modes at $15.5,41.5,43.0$, and $47.5 \mathrm{~cm}^{-1}$ show the opposite behavior.

The phase relationships of the PR experiment have already been shown to provide direct evidence for the determination of molecular motions described by eigenvectors of the Raman active lattice vibrations in TBB. ${ }^{11}$ Thus, the 21.5 and $39.0 \mathrm{~cm}^{-1}$ modes are the $B_{g}$ and $A_{g}$ lattice vibrations having molecular librational motions that are principally inplane, while the other four modes make up the two $A_{g}-B_{g}$ lattice vibration pairs with molecular librational motions that are predominantly out-of-plane.

Turning now to the intramolecular vibrations in TBB, the phases of PR bands for the modes at 125, 203, 208, and $220 \mathrm{~cm}^{-1}$ indicate that their frequencies increase under a compressive stress along [110]*. Except for the $203 \mathrm{~cm}^{-1}$ mode, all of these modes have been assigned as in-plane vibrations..$^{20}$ Thus, consistent with the model used in Ref. 11, the increase of the in-plane intermolecular forces due to the compressive stress is observed to raise the vibrational ener- 
TABLE II. Coupling constants of vibrational modes observed in TBB by piezomodulated Raman spectroscopy. ${ }^{a}$

\begin{tabular}{|c|c|c|c|c|c|c|}
\hline $\begin{array}{l}\text { Mode } \\
\left(\mathrm{cm}^{-1}\right)\end{array}$ & Symmetry & Crystal & $\begin{array}{l}\text { Scattering } \\
\text { geometry }\end{array}$ & $\begin{array}{c}\phi_{\alpha \beta} \\
\left(10^{3} \mathrm{~cm}^{-2}\right)\end{array}$ & $\left|\frac{\phi_{\alpha \beta}}{\omega_{0}^{2}}\right|$ & $\begin{array}{l}\left|\Delta \omega_{0}\right| \\
\left(\mathrm{cm}^{-1}\right)\end{array}$ \\
\hline \multirow{6}{*}{$\begin{array}{c}{[110]^{*} \text { stress, }} \\
15.5\end{array}$} & $e_{6, \mathrm{rms}}=0.0011$ & & & & & \\
\hline & $A_{g}$ & I & $A$ & 0.4 & 1.7 & 0.02 \\
\hline & & & $B$ & 0.57 & 2.4 & 0.029 \\
\hline & & & C & 0.45 & 1.9 & 0.023 \\
\hline & & III & $B$ & 0.18 & 0.8 & 0.009 \\
\hline & & & $C$ & 0.34 & 1.4 & $0.026^{\mathrm{b}}$ \\
\hline \multirow[t]{6}{*}{21.5} & $B_{g}$ & I & $A$ & -3.4 & 7.4 & 0.12 \\
\hline & & & $B$ & -3.2 & 6.9 & 0.12 \\
\hline & & & C & -2.8 & 6.1 & 0.10 \\
\hline & & III & $A$ & -1.7 & 3.7 & 0.062 \\
\hline & & & $B$ & -2.0 & 4.3 & 0.072 \\
\hline & & & C & -2.1 & 4.5 & $0.11^{\mathrm{b}}$ \\
\hline 39.0 & $A_{g}$ & I & C & -1.7 & 1.1 & 0.034 \\
\hline 41.5 & $A_{g}$ & I & $B$ & 1.4 & 0.8 & 0.026 \\
\hline \multirow[t]{2}{*}{43.0} & $B_{g}$ & I & $A$ & 2.3 & 1.2 & 0.041 \\
\hline & & & C & 0.9 & 0.5 & 0.015 \\
\hline \multirow[t]{3}{*}{47.5} & $B_{g}$ & I & $A$ & 2.3 & 1.0 & 0.037 \\
\hline & & & $B$ & 1.4 & 0.6 & 0.022 \\
\hline & & & C & 1.7 & 0.8 & 0.028 \\
\hline 125 & $a_{g}$ & I & C & -2.6 & 0.16 & 0.016 \\
\hline 220 & $a_{g}$ & I & C & -11 & 0.23 & 0.040 \\
\hline 667 & $a_{g}$ & I & C & 11 & 0.03 & 0.013 \\
\hline \multirow{3}{*}{$\begin{array}{c}{[001] \text { stress, }} \\
15.5\end{array}$} & $e_{3, \mathrm{rms}}=0.0008$ & & & & & \\
\hline & $A_{g}$ & II & $D$ & -0.63 & 2.6 & 0.023 \\
\hline & & & E & -0.56 & 2.3 & 0.021 \\
\hline \multirow[t]{2}{*}{21.5} & $B_{g}$ & II & $D$ & 2.4 & 5.2 & 0.064 \\
\hline & & & E & 1.9 & 4.1 & 0.049 \\
\hline 39.0 & $A_{g}$ & II & $D$ & 2.7 & 1.8 & 0.040 \\
\hline 41.5 & $A_{g}$ & II & $D$ & -4.0 & 2.3 & 0.055 \\
\hline 43.0 & $B_{g}^{\circ}$ & II & E & -4.4 & 2.4 & 0.058 \\
\hline 47.5 & $B_{g}^{\circ}$ & II & E & -3.8 & 1.7 & 0.045 \\
\hline
\end{tabular}

${ }^{\mathrm{a}} \phi_{\alpha \beta}$ is the effective coupling constant, $\omega_{0}$ is the mode frequency, and $\left|\Delta \omega_{0}\right|$ is the change in mode frequency at the peak of the stress cycle.

${ }^{\mathrm{b}} e_{6, \mathrm{rms}}=0.0016$.

gies of the modes. The unexpected band shape and the absence of signal for the PR bands corresponding to the 667 and $1122 \mathrm{~cm}^{-1}$ fundamentals, respectively, precludes a determination of the effects of the [110]* stress on their mode frequencies. Nevertheless, it indicates that relative to the other modes, these must be associated with significantly more harmonic behavior.

\section{B. Anisotropic anharmonic coupling constants}

As previously discussed, the intensity of the PR band of a given Raman-active vibration relative to its Raman intensity is a measure of the anharmonicity exhibited by the mode. Curve-fitting procedures can be used to obtain the strain-induced coupling constant, $\phi_{\alpha \beta}$, pertaining to each vibrational mode in a crystal subjected to a particular stress. Since compressive and tensile strains along the directions of the stresses applied in the experiments may be calculated using Eqs. (8) and (9), strains $e_{6}$ and $e_{3}$, which correspond to the $[110]^{*}$ and $[001]$ stresses, ${ }^{22}$ respectively, can be determined. By considering symmetry, the subscripts on the strains may be condensed by employing the well-known Voigt matrix notation. ${ }^{18}$ For convenience, the matrices are defined in the $a^{*} b c$ axial system since the stresses applied in the PR experiments were either perpendicular or parallel to the $\mathrm{c}$ axis in TBB (see Fig. 2). With values for the strains, effective coupling constants $\phi_{6}$ and $\phi_{3}$ can be extracted for each of the vibrational modes measured in the PR spectra of TBB. The values derived for the effective coupling constants are understood to include contributions from any accompanying, undetermined strains corresponding to each of the applied stresses.

The curve-fitting procedure described in Sec. II was used to calculate effective coupling constants from the experimental data. These constants determined from the lattice modes observed in the PR spectra of TBB have been tabulated in Table II. They are shown according to stress direction, scattering configuration, and crystal sample. A negative value for a given constant indicates that the corresponding lattice mode increases in frequency under compressive stress.

The accuracy of the values for the effective coupling constants, based on the limitations of the curve-fitting technique and the calculations of the strains is between $10 \%$ and $20 \%$ except for the values obtained for the modes between 40 and $50 \mathrm{~cm}^{-1}$ under a [110]* stress. The strong overlap in this region of the spectrum and the resultant unusual PR band shapes made it difficult to produce good calculated fits 
to the data. Therefore, these effective coupling constants were estimated by an approximate fit of the peak-to-peak distance in the PR bands for the modes.

In addition to the coupling constant, the magnitude of the frequency shift at the peak of the stress cycle, $\left|\Delta \omega_{0}\right|$, is shown in Table II for each mode. From the definitions

$$
\omega_{+}=\omega_{0}+\left|\Delta \omega_{0}\right|
$$

and

$$
\omega_{-}=\omega_{0}-\left|\Delta \omega_{0}\right|
$$

and using Eqs. (3), (6), and (7) the formula for $\left|\Delta \omega_{0}\right|$ is found to be

$$
\left|\Delta \omega_{0}\right|=\left|\Delta e_{\alpha \beta} \cdot \phi_{\alpha \beta}\right| / 4 \omega_{0}
$$

for small strains. The stress-induced frequency shifts for the vibrations in the TBB crystal are on the order of $0.1 \mathrm{~cm}^{-1}$ or less, which is consistent with the values expected from calculation of the stress Grüneisen parameters for molecular crystals. ${ }^{9,23}$ This is an important aspect of the piezomodulated Raman experiment. The measurement of small frequency shifts such as this would be extremely difficult in a static experiment, whereas the modulated stress applied here allows for a much more accurate differential measurement. Moreover, since such small strains are induced in the crystal, the experiments are performed in the elastic regime under effective pressures of only 10-100 bars. Previous Raman experiments in molecular crystals employing hydrostatic stresses have applied pressures on the order of kilobars in order to observe frequency changes in the vibrational modes.

In answer to a question raised in the theoretical work of Luty and Eckhardt, ${ }^{9}$ it should be noted that the extrema of the PR bands are not located at $\omega_{-}$and $\omega_{+}$. Rather, it is the width of the Raman band, $\gamma$, that tends to dictate their frequency positions. It can be seen from the values of $\left|\Delta \omega_{0}\right|$ obtained for the various modes that, due to the bandpass of the monochromator, it would be impossible to observe a PR band in TBB if its extrema were separated by only $2\left|\Delta \omega_{0}\right|$.

In order to use the derived coupling constants to discuss the extent of anharmonicity of the vibrational modes in TBB, they should also be expressed as a fraction of the quadratic mode frequency. A low energy vibration will be perturbed by coupling of a specified strength to a much greater event than a high energy vibration ${ }^{24}$ and its associated potential energy surface will be more anharmonic. Equation (12) demonstrates that for a given coupling constant and strain, this also means the lower energy modes will undergo the larger frequency shifts. Consequently, these fractions have been included in Table II as well.

It is readily seen from Table II that the $21.5 \mathrm{~cm}^{-1} B_{g}$ lattice vibration in TBB exhibits by far the greatest amount of strain-induced coupling with other modes. By displaying such behavior, it is a good candidate as the anharmonic mode most involved in the structural phase transition mechanism for TBB. The $15.5 \mathrm{~cm}^{-1} A_{g}$ mode is the next most anharmonic vibration, with the remaining lattice modes only somewhat less so. The coupling observed in the lattice vibra- tions is approximately the same for both stress directions. This assessment, however, is less certain for the modes above $40 \mathrm{~cm}^{-1}$ where curve fits for the [110]* stress are not as reliable.

The agreement of the coupling constant values obtained for a given lattice mode and stress direction is quite good when considering data from different scattering geometries for the same crystal sample. Values for the 15.5 and 21.5 $\mathrm{cm}^{-1}$ modes obtained from different crystals (samples I and III) were found to vary more widely. The averaged values from the two different samples generally had about $30 \%$ error in precision. The reason for this probably lies in the sensitivity of the experiment to crystal quality. Any defect in a crystal, such as cracks, dislocations, or vacancies, will weaken the strains induced by an applied stress because the crystal will have additional degrees of freedom available (through the breakdown of translational symmetry and crystal integrity) by which it can accommodate the lower strain energy. Therefore, the crystal samples that exhibit the largest PR responses for a given stress most likely give results more representative of a perfect lattice, since the data indicate they suffer the greater strains. This means piezomodulated Raman spectroscopy also lends itself as a tool in the study of crystal quality, particularly in the detection of microscopic defects.

Effective coupling constants for the intramolecular modes in TBB at 125 and $220 \mathrm{~cm}^{-1}$ are also given in Table II. Constants for the 203 and $208 \mathrm{~cm}^{-1}$ modes were not determined due to their low Raman and PR signals. As expected, a comparison of the $\left|\phi_{\alpha \beta}\right| / \omega_{0}^{2}$ fractions of the 125 and $220 \mathrm{~cm}^{-1}$ modes to those of obtained for the lattice modes reveals that the molecular vibrations are significantly less anharmonic. The assumption that the molecular modes in TBB can be discussed by treating the crystal as an oriented gas appears to be reasonable since separation of the internal and external vibrations is possible. The PR data indicate the in-plane bromine stretch mode at $220 \mathrm{~cm}^{-1}$ in TBB is more anharmonic than the in-plane bromine bend vibration at $125 \mathrm{~cm}^{-1}$. In support of this, the width of the $220 \mathrm{~cm}^{-1}$ band is seen to be more than five times greater than the $125 \mathrm{~cm}^{-1}$ Raman bandwidth.

A coupling constant for the totally symmetric, in-plane ring deformation and bromine stretch at $667 \mathrm{~cm}^{-1}$ could only be estimated from the peak-to-peak intensities of its PR band because of its unusual band shape. This mode and the ring breathing $1122 \mathrm{~cm}^{-1}$ vibration, which exhibited essentially no PR response, are found to undergo even less straininduced PR coupling than the lower frequency molecular modes. Hence, the extent of anharmonicity in the intramolecular vibrations is not only less than in the lattice modes, but also decreases with increasing energy of the molecular mode, as predicted by theory. The vibrational modes arising from covalently bonded forces are not perturbed by intermolecular interactions to the same extent as the lattice modes which derive their restoring forces from van der Waals interactions. 


\section{SUMMARY}

Piezomodulated Raman spectroscopy has been employed to determine strain-induced coupling constants for each of the Raman active lattice modes and a few of the intramolecular vibrations in single crystals of 1,2,4,5tetrabromobenzene. These coupling constants, which are directly related to the first anharmonic term in the potential energy expansion used in lattice dynamical calculations, are a quantitative measurement of mode anharmonicity in the TBB molecular crystal.

Lattice modes, as expected, are significantly coupled by the induced strains and the coupling is dependent on the direction and symmetry of the applied stress. The molecular modes, in accordance with accepted theories, display less coupling due to intermolecular interactions in the crystal lattice and the modes in general show less anharmonicity with increasing frequency.

The phonon mode that exhibits the largest strain-induced coupling is the $21.5 \mathrm{~cm}^{-1} B_{g}$ lattice vibration. Its high degree of anharmonicity is consistent with the fact that it undergoes the greatest frequency change of all the modes as the TBB crystal passes from the beta to the gamma phase during its structural phase transition. The resulting $28 \mathrm{~cm}^{-1}$ mode in the gamma phase has also been shown to exhibit a large PR response. ${ }^{10}$

The research demonstrates that piezomodulated Raman spectroscopy can be used for direct measurement of the anisotropy of the anharmonicity of both inter- and intramolecular vibrational modes. This information, which is rarely determined and then only with difficulty, offers the potential for significant new experimental and theoretical insights into the vibrational behavior of molecules and crystals. Further piezomodulated Raman studies of TBB and other molecular crystals not only will aid in understanding the underlying mechanisms and energetics involved in solid-solid transitions, especially those that are strain-mediated, but will also serve to monitor and characterize solid state reactions.
${ }^{1}$ See, for example, Chem. Mater. 6, No. 8 (1994).

${ }^{2}$ A. J. Pertsin and A. I. Kitaigorodsky, in The Atom-Atom Potential Method: Applications to Organic Molecular Solids, Vol. 43 in Springer Series in Chemical Physics, edited by V. I. Goldanskii, F. P. Schafer, and J. P. Toennies (Springer, Berlin, 1987).

${ }^{3}$ M. Born and K. Huang, Dynamical Theory of Crystal Lattices (Oxford University, London, 1954).

${ }^{4}$ G. Venkataraman, L. A. Feldkamp, and V. C. Sahni, Dynamics of Perfect Crystals (MIT, Cambridge, 1975).

${ }^{5}$ P. Bruesch, in Phonons: Theory and Experiments I: Lattice Dynamics and Models of Interatomic Forces, Vol. 34 in Springer Series in Solid-State Sciences, edited by M. Cardona, P. Fulde, and H.-J. Queisser (Springer, Berlin, 1982).

${ }^{6}$ C. Carlone, C. Cyr, S. Jandl, N. K. Hota, and J. Zauhar, J. Chem. Phys. 77, 4920 (1982).

${ }^{7}$ E. M. Anastassakis, in Dynamical Properties of Solids: Disordered Solids, Optical Properties, edited by G. K. Horton and A. A. Maradudin (NorthHolland, Amsterdam, 1980), Vol. 4.

${ }^{8}$ B. A. Weinstein and R. Zallen, in Light Scattering in Solids IV: Electronic Scattering, Spin Effects, SERS, and Morphic Effects, Vol. 54 in Topics in Applied Physics, edited by M. Cardona and G. Guntherodt (Springer, Berlin, 1984).

${ }^{9}$ T. Luty and C. J. Eckhardt, J. Chem. Phys. 82, 1515 (1985).

${ }^{10}$ K. M. White and C. J. Eckhardt, Phys. Rev. Lett. 59, 574 (1987).

${ }^{11}$ K. M. White and C. J. Eckhardt, J. Chem. Phys. 92, 2214 (1990).

${ }^{12}$ R. A. Cowley, in The Raman Effect, edited by A. Anderson (Marcel Dekker, New York, 1971), Vol. 1, p. 95.

${ }^{13}$ J. Sartwell and C. J. Eckhardt, Phys. Rev. B 48, 13361 (1993).

${ }^{14}$ K. M. White and C. J. Eckhardt, J. Chem. Phys. 90, 4709 (1989).

${ }^{15}$ F. B. Johnson, Nature (London) 178, 590 (1956).

${ }^{16}$ G. Gafner and F. H. Herbstein, Acta Crystallogr. 13, 706 (1960).

${ }^{17}$ G. Gafner and F. H. Herbstein, Acta Crystallogr. 17, 982 (1964).

${ }^{18}$ J. F. Nye, Physical Properties of Crystals (Oxford University, New York, 1985).

${ }^{19}$ K. M. White, K.-H. Brose, and C. J. Eckhardt, J. Chem. Phys. 85, 5551 (1986).

${ }^{20} \mathrm{~K}$. M. White and C. J. Eckhardt (unpublished).

${ }^{21}$ N. B. Colthup, L. H. Daly, and S. E. Wiberley, Introduction to Infrared and Raman Spectroscopy, 2nd ed. (Academic, New York, 1975).

${ }^{22}$ It can be shown that for the TBB unit cell, a uniaxial strain along [110]* is essentially $e_{6}$.

${ }^{23}$ C. L. Choy, S. P. Wong, and K. Young, Phys. Rev. B 29, 1741 (1984).

${ }^{24}$ I. G. Main, Vibrations and Waves in Physics (Cambridge University, Cambridge, 1978). 